\title{
Escala de Felicidad para Adultos (EFPA)
}

\section{Happines Scale for Adults (EFPA)}

\author{
Emilio Moyano Díaz \\ Universidad de Talca, Chile \\ Daniela Dinamarca \\ Universidad de Talca, Chile \\ Rodolfo Mendoza-Llanos \\ Universidad del Bío-Bío, Chile \\ Gonzalo Palomo-Vélez \\ Vrije Universiteit, Holanda
}

Recibido (11/11/2016) Aceptado (23/03/2018)

Agradecimientos: Estudio posible gracias al financiamiento del Proyecto Fondecyt $\mathrm{N}^{\circ} 1131152$. Grupo de Investigación en Calidad de Vida y Ambientes Saludables (GICVAS), Facultad de Psicología, Universidad de Talca (Chile).

Correspondencia: Emilio Moyano Díaz, 2 Norte 685, Talca, Chile, email: emoyano@utalca.cl 


\title{
Resumen
}

Estudiar la felicidad psicométricamente es reciente, y los escasos instrumentos disponibles son de raíz inglesa. Felicidad es un concepto no consensuado aún, típicamente medido como unidimensional, mediante pocos ítems directos, y generalmente sin especificarse qué lo constituye. En el Estudio 1 y basados en un concepto pentadimensional y emic de felicidad, fue construida una escala de 100 ítems para medirla en adultos chilenos. Aplicada a diferentes muestras $(n=68 ; n=277)$ y mediante análisis factorial exploratorio fue depurada, dando origen a la Escala de Felicidad Para Adultos (EFPA) -con 21 ítems- de buena confiabilidad y validez, distribuidos en cuatro dimensiones: estado psicológico, tener familia, orientación de logro y optimismo. En un Estudio 2 fue realizada una validación cruzada de la EFPA en una nueva muestra de adultos ( $\mathrm{n}=341)$, y mediante análisis paralelo y ecuaciones estructurales se probó diferentes modelos, confirmándose uno de cuatro y otro de tres dimensiones, optándose por éste último: estado, tener familia y orientación de logro Palabras clave: felicidad, medición, estructura, validez.

\begin{abstract}
Studying happiness psychometrically is recent, and the few available instruments are English rooted. Happiness is a concept that hasn't reached to an agreement yet, typically measured as unidimensional, through a few direct items, and usually not specifying what constitutes it. In study 1, and based on a pentadimensional and emic concept of happiness, a 100 items scale was built to measure it among Chilean adults. It was applied to different samples $(n=68 ; n=277)$ and refined through exploratory factor analysis, giving origin to the Happiness Scale for Adults (EFPA) - composed by 21 items- with good reliability and validity, distributed among four dimensions: psychological state, having family, achievement orientation and optimism. In study 2 , the EFPA crossed validity was carried out with a new sample of adults ( $\mathrm{n}=341)$, and through parallel analysis and structural equation modelling various models were tested, being confirmed one of 4 and other of 3 dimensions, keeping the later: state, having family and achievement orientation.

Keywords: happiness, measure, structure, validity.
\end{abstract}

\section{Introducción}

La felicidad es tema de antigua preocupación filosófica que recientemente alcanza interés científico en psicología (Carr, 2004; Haidt, 2007; Seligman, 2004). A diferencia del concepto aristotélico de felicidad centrado en la eudaimonía y virtud, que ha sido recogida en el concepto de bienestar psicológico (BP) (Diener, 2000; Kashdan, Biswas-Diener, y King, 2008; Ryan y Deci, 2001), en psicología la felicidad es prevalecientemente concebida desde perspectiva hedónica, entendiéndole como la presencia e intensidad de emociones o sentimientos positivos, y se le ubica generalmente como componente emocional del constructo bienestar subjetivo (BS). El debate entre estas dos corrientes filosóficas -eudamonía y hedonismo- ha tenido su derivación a la psicología de la calidad de vida, del bienestar y de la felicidad. Desde la eudamonia se propone alcanzar la felicidad a través del virtuosismo, mediante la realización del potencial personal de una vida dotada de sentido y espiritualidad, e inspiró el concepto de bienestar psicológico (BP). Desde el hedonismo en cambio se propone la búsqueda del bienestar y felicidad a través del placer, del vivir satisfaciendo las necesidades y evitando el dolor (Ryan y Deci, 2001). Más específicamente, para Lyubomirsky (2001; Lyubomirsky y Lepper, 1999) la felicidad es un nivel relativamente estable de experiencias de bienestar en un período específico de tiempo ( 3 o 6 meses). Bekhet, Zauszniewski y Nakhla (2008) plantean que la felicidad refiere a un sentimiento positivo que todas las personas quieren alcanzar, siendo un atributo construido socialmente, y que varía de un lugar a otro y de una persona a otra. Así, representa un desafío tratar de desentrañar culturalmente su significado, y especialmente al constatarse que las escasas definiciones utilizadas provienen de culturas diferentes a la latinoamericana primando en ellas la perspectiva hedónica de Diener (1994). Lyubomirsky y Lepper (1999) en esta línea entienden que la felicidad, aunque tradicionalmente 
ha sido estudiada en relación a variables denominadas 'objetivas' que influencian el bienestar, deriva sobre todo de la percepción subjetiva de aquellos eventos y de su impacto en el bienestar.

Hay quienes proponen tres componentes fundamentales para el desarrollo de la felicidad: 1.- la frecuencia de emociones positivas y estados de ánimo positivos. 2.- bajos niveles de sentimientos y emociones negativas y 3.- la estabilidad temporal del grado de satisfacción personal (Francis, 1999; Pannells y Claxton, 2008; Stewart, Watson, Clark, Ebmeier, y Deary, 2010). Así, en las personas operarían dos procesos, uno emocional -la experiencia- y uno cognitivo -la valoración de la primera-, de modo que aquellas comprenden y valoran lo que experimentan, haciendo de la experiencia de la felicidad una evaluación subjetiva (Diener, 1994). Considerando estos componentes y su eventual predominio Venhoveen (2006) clasificó las definiciones de felicidad en cuatro tipos: afectivas (v.g. Bradburn, 1969; Fordyce, 1971), actitudinales (Veenhoven, 1994), cognitivas (Shin y Johnson, 1978: 478), y mixtas (Chekola, 1974). Los juicios acerca de la felicidad incluyen autoevaluaciones afectivas globales y clasificación de afectos, todo lo cual es esencialmente subjetivo (Myers y Diener, 1995).

Revisiones recientes evidencian que en Latinoamérica las investigaciones acerca de felicidad están focalizadas en medir este constructo más que en su análisis teórico o conceptual (Moyano Díaz, 2016). Y sin embargo, en ciencia lo más importante son las teorías y conceptos rigurosos, claros y distintos que las constituyen en redes lógicamente conectadas. Someter a análisis y definir el concepto de felicidad se torna aún más relevante dada la falta de consenso acerca de su definición y, más aún, que para algunos se trata de una categoría en construcción (Silva-Colmenares, 2008). Las distinciones entre los conceptos de calidad de vida, bienestar y felicidad, "son todavía confusas y, consecuentemente, su operacionalización en términos de medirlos se muestra muchas veces deformada" (Scorsolini-Comin y dos Santos, 2010, p.4; Gómez y Villegas de Posada, Barrera y Cruz, 2007). En autores latinoamericanos encontramos tres aportaciones a la construcción del concepto de felicidad. Alarcón (2006) afirma que no habría significados diferentes en lengua española ni en el habla peruana cotidiana entre bienestar y felicidad, aun reconociendo que éste último es semánticamente más complejo y rico que el primero. No obstante esto, se suma al enfoque hedónico y a Diener, optando por sinonimizar bienestar y felicidad definiendo ésta como "un estado afectivo de satisfacción plena que experimenta subjetivamente el individuo en posesión de un bien anhelado" (2006, p.101). Desde un análisis factorial propone que la felicidad estaría constituida por cuatro factores: ausencia de sufrimiento profundo, satisfacción con la vida, realización personal y alegría de vivir.

Álvarez-Ramirez propone un concepto multidimensional de felicidad, y desde un análisis acerca de creencias respecto de ésta concluyó que "la felicidad es una representación diversa, heterogénea, borrosa y múltiple de emociones, sensaciones, sentimientos, actitudes, valores, motivaciones y experiencias bio-psico-socio-culturales que se entrelazan, vinculándose en lo individual, en el vínculo con los demás y con la percepción del mundo o la vida misma del sujeto" (2012, p.325).

Más recientemente y mediante enfoque cualitativo y 'emic', hemos construido una definición de felicidad desde el contenido de las respuestas que adultos dieron a la pregunta ¿Qué es para Ud. la felicidad? La definición propuesta es: "Un estado más o menos transitorio de equilibrio, relativo a condiciones, resultados o logros individuales o grupales favorables o reconfortantes para la persona, generalmente asociados a una sensación de satisfacción y bienestar. Este estado surge con base a la estabilidad de ciertos elementos relevantes para la persona, de seguridad y sostén básico en lo económico (trabajo) y familiar, que le posibilitan dedicarse con libertad a actividades que implican posibilidades de goce, desarrollo y plenitud" (Moyano Díaz, 2016). El análisis de las respuestas permitió construir el 'pentágono de la felicidad' conformado por las 5 dimensiones siguientes: Estado, Estar, Tener, Logro y Bienestares.

Dos de las tres definiciones referidas por autores latinoamericanos han sido construidas mediante enfoque etic, $\mathrm{y}$ una emic, pero todas convergen en proponer un concepto multidimensional o complejo de felicidad.

\section{Instrumentos para medir felicidad.}

Las escalas para medir felicidad actualmente en uso son aportaciones útiles, generalmente del tipo lápiz y papel, de origen anglosajón y para adultos: la Escala de Felicidad Subjetiva (EFS) de Lyubomirsky y Lepper (1999), la de Felicidad de la Universidad de Memorial de Newfoundland (MUNSH) (Kozma y Stones, 1980) y el Inventario de Felicidad de Oxford (OHI, de Argyle, Martin y Crossland, 1989). Estos instrumentos tienen diferentes estructuras de composición (la EFS de cuatro ítems, monodimensional, la MUNSH de 24 ítems, hexadimensional y la OHI de 29 
ítems, tetradimensional), y todas cumplen con propiedades psicométricas adecuadas (EFS: alfa media $=0.86$; MUNSH $>0.85$; OHI $=0,58$, Ortiz, Gancedo, y Reyna, 2013). Existen adaptaciones para Chile de la EFS (alfa $=0.79$, Moyano Díaz y Ramos, 2007; alfa $=$ de 0.73 a 0.87 Vera-Villarroel, CelisAtenas y Córdova-Rubio, 2011) y de la MUNSH (alfa $>0.90$, Moyano Díaz, Flores, y Soromaa, 2011). La validez de la EFS es muy cuestionable, ya que ella no recoge ni informa qué entienden por felicidad quienes responden, quedando este concepto solo implícito. Adicionalmente, hay al menos un reporte de aplicación de la EFS en sectores populares en Chile que evidencia baja confiabilidad (alfa de Cronbach 0.55 ), dónde los ítems números 4 y 5 presentan serias dificultades de comprensión para esas personas (Hernández y Muñoz, 2016).

En lo mejor de nuestro conocimiento, hay autores latinoamericanos que han ido más allá que la mera adaptación $\mathrm{y}$ aplicación de instrumentos anglosajones en la región y han creado instrumentos propios para medir felicidad. Se trata de Alarcón (2006) en Perú, Rojas (2007) en México, y Álvarez Ramírez (2012) en Colombia.

Alarcón (2009) construyó la Escala de Felicidad de Lima (EFL) de 27 ítems en formato de respuesta likert de 5 puntos, incluyendo ítems de las escalas de felicidad de Oxford (Argyle et al., 1989; Hills y Argyle, 2002), de la Escala de Satisfacción Vital de Diener et al. (1985) más otros de creación propia. Reportó una fiabilidad alfa de Cronbach de 0.91 y cuatro factores explicativos del $49.79 \%$ de la varianza total. Una adaptación de la EFL con 14 ítems a adultos mayores venezolanos ( $\mathrm{n}=103$, de 60 a 85 años) arrojó una consistencia interna de 0.84 , reproduciéndose su composición factorial de cuatro factores explicativos -en este caso- del 61.38\% de la varianza (Árraga y Sánchez, 2012). Las cuatro dimensiones de la escala son: 1.-Sentido positivo de la vida: relacionada con los sentimientos positivos y la ausencia de pesimismo e intranquilidad. 2.- Satisfacción con la vida: refiere al grado de satisfacción que expresa la persona con su propia vida, ésta satisfacción es apreciada por la percepción que tenga la persona de haber alcanzado las metas que se ha propuesto en la vida. 3.- Realización personal: refleja la tranquilidad emocional de la persona basada en la orientación hacia las metas que considera que son importantes para su vida. 4.- Alegría de vivir: referida a la expresión de la felicidad a través de la alegría y el optimismo, es pensar positivamente respecto a lo que depara el futuro (Alarcón, 2009).
Rojas (2007) creó un instrumento para identificar el referente de la felicidad que las personas tienen, a partir de ocho diferentes escuelas filosóficas clásicas, de las que derivó ocho frases-afirmación de síntesis respecto del significado de felicidad. Quien responde debe elegir una de las frases que mejor represente su noción de felicidad (referente). Los ocho referentes son: estoicismo, virtud, disfrute, carpe diem, satisfacción, utopía, tranquilidad, autorrealización. De una muestra de 1540 mexicanos el $24.2 \%$ eligió el referente satisfacción - 'la felicidad es estar satisfecho con lo que yo tengo y lo que soy'-, seguido por el estoicismo y el disfrute $-14.6 \%$ - 'la felicidad es aceptar las cosas como ellas son', $14 \%$ 'la felicidad es disfrutar lo que uno ha alcanzado en la vida', respectivamente, y la auto-realización con $11.7 \%$ 'felicidad es ejercer completamente nuestras capacidades', quedando los restantes cinco con menos de $8.2 \%$ cada uno. Mediante su aplicación ha contribuido a explicar la heterogeneidad (inconsistencia) de resultados relativos a la relación ingresos y felicidad (Rojas, 2007).

Álvarez-Ramirez (2012) diseñó -con perspectiva multidimensional- una escala de 47 ítems tipo Likert de creencias acerca de la felicidad. En su estudio de 600 adultos colombianos equi-repartidos por sexo, de 19 a 40 años, controló nivel socioeconómico y buscó caracterizar las dimensiones psicológicas subyacentes a las creencias acerca de la felicidad controlando edad, género y estrato socioeconómico. Reportó un alfa Cronbach total de 0.88 y por factor, entre: $0.88 \leq \alpha \geq 0.78$ más una rotación varimax de ejes principales que arrojó una solución de nueve factores que explicaron el $92 \%$ de la varianza en 47 ítems. Las correlaciones ítem-factor fueron altas: $r=(0.53-0.71)$ e ítem-test: $r=(0.59-0.70)$. Las dimensiones reportadas evidenciarían que la felicidad es entendida de manera diversa, los valores altos de las correlaciones ítem-factor de 0.53 a 0.71 lo ratifican, así como las altas correlaciones ítem-test entre 0.59 a 0.70 con afecto positivo, es decir, el ánimo, la alegría (p. 326).

Las escalas EFL de Alarcón (2006) así como la de creencias acerca de la felicidad de Álvarez-Ramirez (2012) confirman estructuras multidimensionales del concepto de felicidad, lo que es convergente con la definición de Moyano-Díaz (2016) proveniente de enfoque emic. La felicidad aparece así como un atributo construido socialmente, que varía entre personas y de una cultura a otra, cuya definición por tanto es idiosincrática, y es en función de aquella que cada quien se auto-reporta como más o menos feliz (Bekhet, 
Zauszniewski, y Nakhla, 2008). Aunque la felicidad en las definiciones latinoamericanas referidas es concebida multidimensionalmente, lo predominante en los estudios realizados sin embargo es medirle mediante instrumentos unidimensionales, y anglosajones. Probablemente ello se deba por una parte, a la economía que representa el uso de instrumentos breves que permiten mediciones masivas en períodos muy breves de tiempo y, por otra, que se trata de estudios en los cuales la felicidad no constituye necesariamente el tema central sino una variable más (frecuentemente de salida) entre otras que sí constituyen el centro de la investigación (Moyano Díaz, 2016).

Sobre la base de la definición multidimensional - pentagonalde la felicidad para adultos derivada de la investigación previa de enfoque emic (Moyano-Díaz, 2016a), hemos construido un instrumento psicométrico para medir felicidad cuyo proceso de elaboración y depuración ha sido llevado a cabo mediante dos estudios que expondremos a continuación.

\section{Estudio 1}

Se describe el proceso de construcción de una primera escala chilena para medir felicidad (ECHF), cuyas propiedades psicométricas son calculadas a partir de su aplicación a una muestra de estudiantes universitarios.

\section{Método}

\section{Participantes}

Dos cientos setenta y siete estudiantes universitarios de ambos sexos (52\% mujeres) elegidos mediante un muestreo no probabilístico por conveniencia, aceptaron voluntariamente participar del estudio, respondiendo a una invitación general efectuada vía correo electrónico a todos los estudiantes de una universidad del Estado.

\section{Instrumentos}

Son aplicados tres instrumentos aquí, una escala (ECHF) conformada por los ítemes creados para medir felicidad, la Escala de Felicidad Subjetiva (Lyubomirsky y Lepper, 1999), y la Escala de Desesperanza (BHS) de Beck, Weissman, Lester y Trexler (1974).

ECHF: inicialmente conformada por 100 ítemsafirmaciones que se responden en formato Likert de 5 puntos $(1=$ Totalmente en Desacuerdo a $5=$ Totalmente de Acuerdo), ubicables en las 5 dimensiones extraídas y propuestas como el 'pentágono de la felicidad': "Estado" (18 ítems), "Estar" (14 ítems), "Tener" (24 ítems), "Logro" (15 ítems) y "Bienestares" (29 ítems). 26 ítems han sido redactados de forma invertida por lo que se requiere revertir su puntuación para su análisis.

Escala de Felicidad Subjetiva (EFS) (Lyubomirsky y Lepper, 1999, en versión de Moyano-Díaz, 2010): mide felicidad subjetiva global, mediante 4 ítems con formato de respuesta de tipo likert de 7 puntos. Presenta adecuados índices de confiabilidad, mostrando una consistencia interna Alfa de Cronbach de 0.79 y estabilidad test-retest de 0.77 en estudiantes universitarios.

Escala de Desesperanza (BHS) (Beck, Weissman, Lester y Trexler, 1974): mide las expectativas negativas que tienen las personas sobre su futuro, su bienestar y su capacidad para superar las dificultades. Se usa la versión castellana de Mikulic, Cassullo, Crespi y Marconi (2009), constituida por 20 ítems de tipo verdadero y falso, agrupados en 3 dimensiones: afectiva, motivacional y cognitiva.

\section{Procedimiento y plan de análisis}

Fueron creados 100 ítems con formato de respuesta tipo Likert de cinco puntos para conformar la ECHF utilizando como insumo central el modelo teórico 'pentágono de la felicidad' (Moyano-Díaz, 2016a). Para la redacción se utilizó la pauta propuesta por Tornimbeni, Pérez y Olaz (2008) tratando de que todos los ítems fueran congruentes con el constructo a medir, con una extensión adecuada, evitando ambigüedades, y utilizando redacciones apropiadas al nivel educacional de la muestra. Los ítems fueron revisados por 3 expertos en el tema, quienes sugirieron eliminar algunos de ellos (ítems 29, 66, 75 y 89) por tener propiedades de universalidad o redundancia.

Así, la ECHF constituida por 96 ítems fue aplicada a una primera muestra $(\mathrm{N}=68)$ para determinar la relación ítem-test y observar su estructura factorial. El método de extracción utilizado -tanto para este AFE inicial como los siguientes- fue el de mínimos cuadrados no ponderados, ya que simplifica la matriz de correlación observada, haciendo más simple su análisis e interpretación, optándose por el método de rotación Oblimin directo, ya que la correlación entre los ítems fue mayor a 0.32. Seguidamente la escala fue aplicada a una muestra definitiva de $\mathrm{n}=277$ y fueron efectuados AFE para cada dimensión del modelo teórico para purificar su medición, y luego verificar la estructural factorial y validez de la escala. 


\section{Resultados}

\section{Fiabilidady validez de la ECHF}

La aplicación inicial de los 96 ítems de la escala a la muestra piloto arrojó un coeficiente de alfa de Cronbach excelente de 0.97 , y no se observó problemas de comprensión de ítems. Luego, utilizando la ECHF en la segunda muestra $(\mathrm{n}=277)$ se llevó a cabo un AFE obteniéndose un KMO de $0.918(p<0.001)$, dando cuenta de la pertinencia del análisis y arrojando 21 factores explicativos del $61 \%$ de la varianza total. Luego, y por parsimonia, se procedió a la eliminación de los ítems menos representativos de cada factor, utilizando como criterios cargas factoriales menores a 0.45 y consistencias internas (alfa de Cronbach) menor a 0.45 disminuyendo así, a 45 ítems. Este procedimiento fue iterado 5 veces conformándose una escala de 25 ítems, con un ajuste bajo e inadecuada confiabilidad. Debido a ello, se optó por realizar un AFE por cada dimensión teórica según el modelo del 'pentágono de la felicidad' (Moyano-Díaz, 2016a), para identificar los ítems más representativos de cada dimensión y fortalecer la consistencia interna del instrumento.

\section{Validez factorial por dimensiones}

Se llevó a cabo un AFE forzado a 1 factor para los 16 ítems de la dimensión Estado, para los 14 ítems de la dimensión Estar, los 23 de la dimensión Tener, los 14 de la dimensión Logro y los 29 ítems de la dimensión Bienestares. Buscando reducir la extensión de la escala, se procedió a la eliminación de ítems menos representativos o poco fiables, siguiendo para ello el mismo criterio inicial -carga factorial y alfa de Cronbach $<0.45$, quedando un instrumento de 37 ítems. Finalmente, se llevó a cabo dos AFE consecutivos para verificar si este instrumento mantenía la estructura propuesta en Moyano-Díaz (2016a). El primero de éstos mostró 6 factores explicando el $57.86 \%$ de la varianza total y, tal y como en cálculos precedentes fue aplicado el criterio de eliminación de ítems menos representativos -14- quedando 23 ítems en la escala. Estos fueron sometidos al segundo $\mathrm{AFE}(\mathrm{KMO}=0.92$, $p<0.001)$ y posterior depuración de reactivos, obteniéndose un instrumento final de 21 ítems. Este instrumento final tiene alfas de Cronbach adecuados: global (0.93) y por dimensiones (desde 0.76 a 0.90 ), y el AFE arroja 4 factores que explican un $58.7 \%$ de la varianza (ver tabla 1 ).

Tabla 1. Estructura Factorial de ECHF (21 items).

\begin{tabular}{|c|c|c|c|c|}
\hline Ítems & $\begin{array}{c}\text { Dimensión } \\
\text { Original }\end{array}$ & $\begin{array}{c}\text { Carga } \\
\text { Factorial }\end{array}$ & $\begin{array}{l}\text { Varianza } \\
\text { Explicada }\end{array}$ & $\begin{array}{c}\text { Alfa de } \\
\text { Cronbach }\end{array}$ \\
\hline \multicolumn{5}{|l|}{ Factor 1: Estado } \\
\hline 98. No me siento alegre la mayoría de las veces. & Bienestares & 0.783 & \multirow{9}{*}{$39.52 \%$} & \multirow{9}{*}{0.902} \\
\hline 83. No me siento pleno(a). & Bienestares & 0.761 & & \\
\hline 41. No me siento en equilibrio. & Estado & 0.713 & & \\
\hline 88. No estoy contento(a) la mayor parte del tiempo. & Bienestares & 0.699 & & \\
\hline 16. No me siento en armonía conmigo mismo(a). & Estado & 0.683 & & \\
\hline 46. No me siento bien de ánimo. & Estado & 0.677 & & \\
\hline 96. No pienso de manera positive la mayor parte del tiempo. & Bienestar & 0.604 & & \\
\hline 65. No me siento en paz con mi entorno. & Bienestar & 0.581 & & \\
\hline 26. No me siento bien mentalmente. & Estado & 0.566 & & \\
\hline \multicolumn{5}{|l|}{ Factor 2: Tener Familia } \\
\hline 68. Tengo una familia unida. & Tener & 0.923 & \multirow{6}{*}{$10.02 \%$} & \multirow{6}{*}{0.906} \\
\hline 79. Tengo una familia bien constituida. & Tener & 0.820 & & \\
\hline 91. Tengo el apoyo de mi familia. & Tener & 0.797 & & \\
\hline 86. Tengo una familia que se encuentra segura y protegida. & Tener & 0.784 & & \\
\hline 21. Me siento en armonía con mi familia & Estado & 0.736 & & \\
\hline 49. He logrado el reconocimiento de mi familia & Logro & 0.503 & & \\
\hline
\end{tabular}




\begin{tabular}{|c|c|c|c|c|}
\hline Ítems & $\begin{array}{c}\text { Dimensión } \\
\text { Original }\end{array}$ & $\begin{array}{c}\text { Carga } \\
\text { Factorial }\end{array}$ & $\begin{array}{c}\text { Varianza } \\
\text { Explicada }\end{array}$ & $\begin{array}{c}\text { Alfa de } \\
\text { Cronbach }\end{array}$ \\
\hline \multicolumn{5}{|l|}{ Factor 3: Logro } \\
\hline 19. He cumplido con los objetivos que me he propuesto. & Logro & 0.702 & \multirow{3}{*}{$5.87 \%$} & \multirow{3}{*}{0.786} \\
\hline 9. He cumplido con los proyectos que me he propuesto en la vida. & Logro & 0.665 & & \\
\hline 17. Estoy satisfecho con las decisiones que he tomado en la vida. & Estar & 0.592 & & \\
\hline \multicolumn{5}{|l|}{ Factor 4: Optimismo } \\
\hline 36. Creo que me irá bien en el futuro & Estado & 0.633 & \multirow{3}{*}{$3.24 \%$} & \multirow{3}{*}{0.766} \\
\hline 81. Pienso que todo me saldrá bien. & Estado & 0.555 & & \\
\hline 67. Pienso que me he desarrollado como persona & Estar & 0.541 & & \\
\hline
\end{tabular}

Exploración de la asociación de los factores de la ECHF con la EFS y la BHS

Con objeto de analizar la validez de construcción de la ECHF fueron realizados análisis correlacionales (Pearson) de convergencia y divergencia de los factores extraídos de la ECHF con la EFS y las dimensiones de la BHS. Como se puede observar en la tabla 2, todos los factores de la ECHF se asociaron de manera positiva y significativa con la felicidad subjetiva global (EFS), siendo el factor 1 referido principalmente al estado de paz y armonía-, el que mostró la relación más acentuada. Se observan asociaciones significativas y negativas entre los factores de la ECHF y la BHS, a excepción del segundo factor referido a familia de aquella, que no se asoció significativamente con ninguna de las dimensiones de la BHS.

Tabla 2. Correlaciones entre los factores de la ECHF y EFS y las dimensiones de la BHS.

\begin{tabular}{lcccc}
\hline & $\begin{array}{c}\text { ECHF } \\
\text { Estado }\end{array}$ & $\begin{array}{c}\text { ECHF } \\
\text { Tener } \\
\text { Familia }\end{array}$ & $\begin{array}{c}\text { ECHF } \\
\text { Logro }\end{array}$ & $\begin{array}{c}\text { ECHF } \\
\text { Optimismo }\end{array}$ \\
\hline $\begin{array}{l}\text { Escala de Felici- } \\
\text { dad Subjetiva }\end{array}$ & $0.68^{* *}$ & $0.40^{* *}$ & $-0.45^{* *}$ & $0.53^{* *}$ \\
$\begin{array}{l}\text { BHS Dimensión } \\
\text { Afectiva }\end{array}$ & $-0.22^{* *}$ & -0.13 & $-0.14^{*}$ & $-0.39^{* *}$ \\
$\begin{array}{l}\text { BHS Dimensión } \\
\text { Motivacional }\end{array}$ & $-0.42 * *$ & -0.09 & $-0.19^{* *}$ & $-0.46^{* *}$ \\
$\begin{array}{l}\text { BHS Dimensión } \\
\text { Cognitiva }\end{array}$ & $-0.28^{* *}$ & -0.00 & $-0.17 * *$ & $-0.28^{* *}$ \\
\hline
\end{tabular}

Nota $* \mathrm{p}<0.05 ; * * \mathrm{p}<0.01$
Se deduce cuatro factores para la ECHF el primero de los cuales denominamos Estado (9 ítems) referido a la felicidad como estado mental de armonía, tranquilidad, paz interior y equilibrio con el medio ambiente. El segundo -Tener familia (6 ítems), referido a contar con una familia unida, que apoya, segura y protegida, y en armonía y reconocimiento. El tercero - Orientación al logro- (3 ítems) referido a haber conseguido los objetivos y proyectos propuestos, y la satisfacción con las decisiones tomadas. El cuarto - Optimismo- (3 ítems), referido a un estilo cognitivo positivo hacia el futuro así como a una evaluación retrospectiva positiva. La confiabilidad obtenida para ella es buena -tal como ha sido mencionado anteriormente- y respecto a la validez de constructo los análisis muestran que efectivamente -y según lo esperadocada uno de los factores de la ECHF están correlacionados positivamente con la EFS (entre 0.40 y 0.68 ) y todos menos uno-familia- negativamente con la BHS (entre -0.14 y -0.46) afirmando la validez de construcción de la misma.

\section{Estudio 2}

El propósito aquí es verificar las propiedades psicométricas de la ECHF en una nueva muestra independiente, lo cual se realiza mediante un procedimiento de validación cruzada. Hemos decidido cambiar su nombre a Escala de Felicidad para Adultos (EFPA).

\section{Método}

\section{Muestra}

Conformada por 341 adultos de ambos sexos ( $71.5 \%$ mujeres), funcionarios de diferentes estamentos de dos hospitales 
públicos ubicados en la región centro sur de Chile. Sus edades fluctuaron entre 24 años o menos (3\%) hasta $55 \mathrm{o}$ más (13.1\%).

\section{Procedimiento}

El EFPA final obtenido del Estudio 1 fue aplicado individualmente a una muestra independiente de 341 trabajadores de dos hospitales públicos de la región del Maule en sus lugares de trabajo, a lo largo de tres semanas. Se utilizó cuatro estrategias para evaluar la validez de contenido: Análisis Paralelo de Horn y Análisis Factorial Confirmatorio (AFC) mediante ecuaciones estructurales; validez convergente mediante significancia estadística de los coeficientes estandarizados; validez discriminante mediante correlaciones inter-factores; y cálculo de estimadores para la consistencia interna.

El análisis paralelo es uno de los métodos más recomendados para determinar el número de componentes presentes, que compara los valores propios observados con los de las variables normales no correlacionadas calculados mediante método de Monte-Carlo, esto es, un factor se mantiene si el valor propio asociado es mayor que el percentil 95 de la distribución de valores propios derivados de los datos aleatorios (Gómez et al, 2016).

En el AFC los modelos fueron estimados mediante mínimos cuadrados no ponderados con ajuste en medias y varianzas (WLSMV). La bondad de ajuste fue evaluada mediante el índice de ajuste comparativo (CFI), el índice de TuckerLewis (TLI) y el error cuadrático medio de aproximación (RMSEA). Valores de CFI y TLI superiores a 0.90 y 0.95 , y valores de RMSEA entre 0.08 y 0.05 , se consideran aceptables y buenos respectivamente (Schermelleh-Engel, Moosbrugger y Müller, 2003). Todos los análisis se realizaron mediante MPlus v. 7.

La validez de la escala se calculó en términos de consistencia interna por medio de los siguientes coeficientes: Alfa de Cronbach, Alfa Ordinal, Omega de McDonald y Theta de Armor. El Alfa de Cronbach asume una naturaleza continua de las variables que no es propia de las escalas tipo Likert, siendo entonces lo pertinente utilizar estimadores para escalas ordinales de datos. Así, el cálculo es efectuado utilizando matrices de correlaciones policóricas mediante el software libre FACTOR (Elosua y Zumbo, 2008), y la hoja de cálculo Excel de Domínguez (2012).

\section{Resultados}

\section{Validez de contenido}

El análisis paralelo indica que los primeros tres valores propios fueron superiores a los generados aleatoriamente para los 21 ítems, mientras que del cuarto en adelante los valores propios observados fueron más bajos, de tal manera que orientan un AFC de tres factores (Fig. 1). Aun así, y para evaluar el instrumento tal cual fue propuesto en el estudio 1, se realiza también AFC para los cuatro factores.

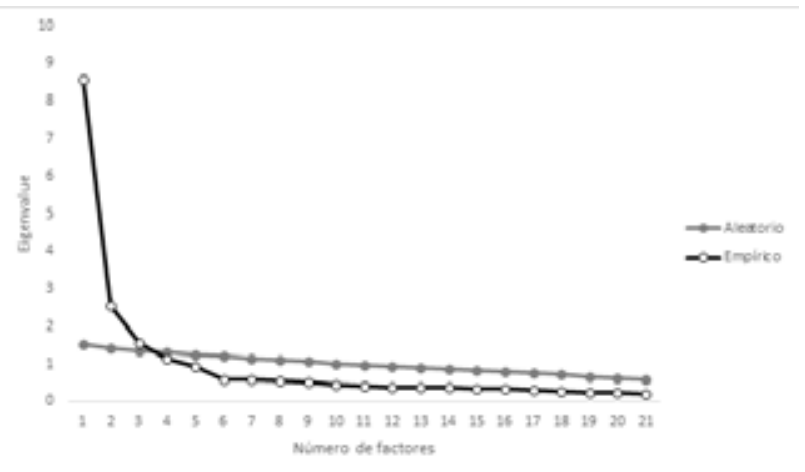

Figura 1. Resultados del análisis paralelo con valores propios observados y aleatorios

Como se puede observar en la tabla 3 tanto el modelo de cuatro factores como el de tres estimados sobre la muestra obtuvieron índices de ajuste adecuados (CFI=0.979; $\mathrm{TLI}=0.976$; RMSEA $=0.070$ y $\mathrm{CFI}=0.976$; $\mathrm{TLI}=0.973$; RMSEA $=0.074$ respectivamente), lo que significa que ambos son válidos. En ambos modelos los ítems explican entre un 55.2 y un $90.1 \%$ de la varianza, por lo cual los ítems se pueden mantener -sin eliminarse ninguno- en ambos modelos. 
Tabla 3. Cargas factoriales y correlaciones según AFC para los dos modelos especificados

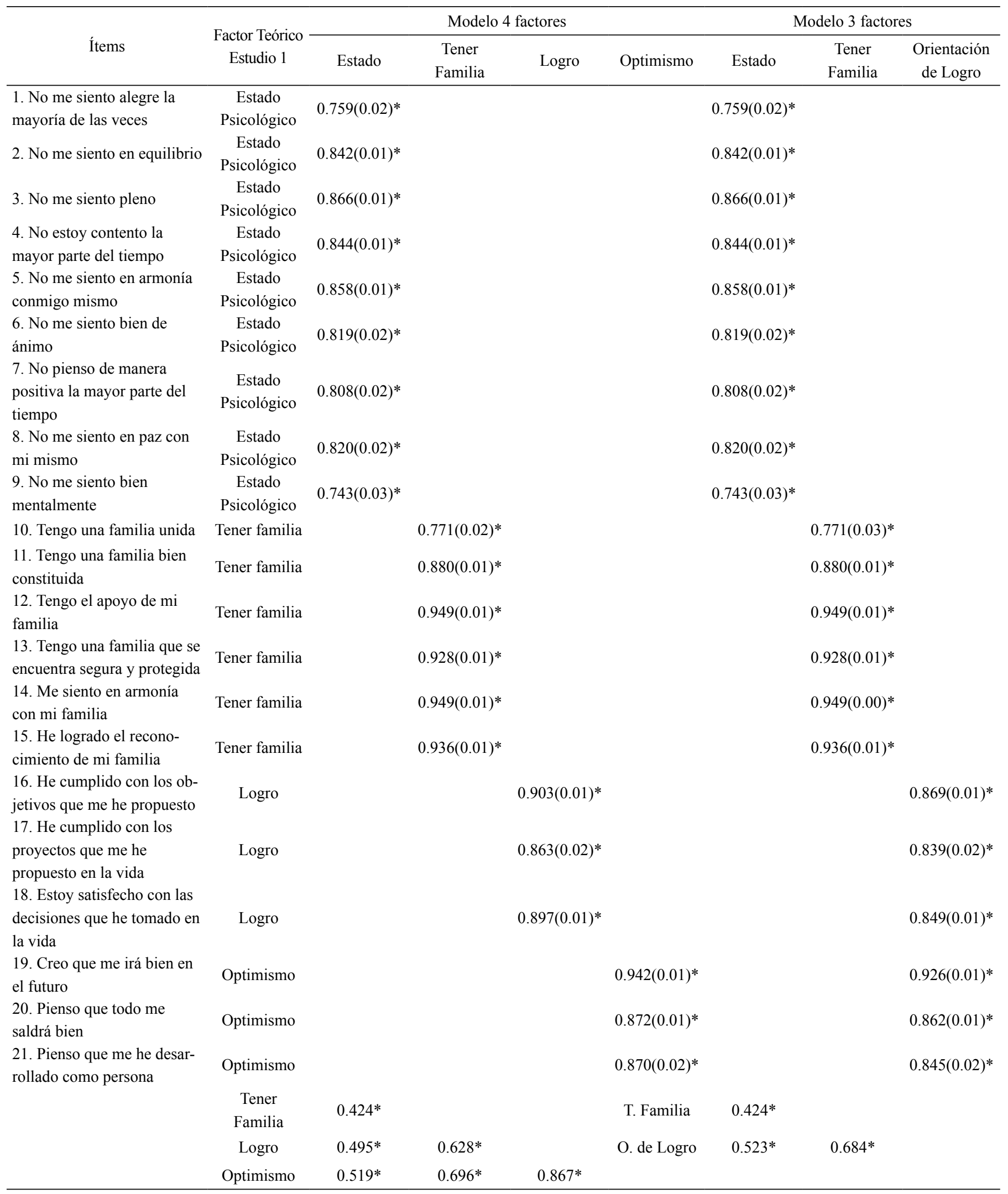

Nota. Valores entre paréntesis corresponden al error estándar.* $\mathrm{p}<0.01$ 
Todos los coeficientes de consistencia interna para el instrumento de tres dimensiones son adecuados (Tabla 4). Theta asume un análisis factorial (método de componentes principales) en tanto Omega es una medida de fiabilidad de una prueba de homogeneidad basada en el modelo de un solo factor, por lo que es especialmente adecuada para evaluar la fiabilidad de cada uno de los tres factores de la escala. Aun cuando el Alfa de Cronbach es más bajo en todos los factores que el Alfa Ordinal, las variaciones generales están entre 0.904 (Logro) y 0.965 (Tener familia).

Tabla 4. Coeficiente de consistencia interna para la versión final del instrumento

\begin{tabular}{lccc}
\hline & $\begin{array}{c}\text { Estado } \\
\text { Psicológico }\end{array}$ & $\begin{array}{c}\text { Tener } \\
\text { Familia }\end{array}$ & $\begin{array}{c}\text { O. de } \\
\text { Logro }\end{array}$ \\
\hline Alfa de Cronbach & 0.911 & 0.927 & 0.904 \\
Alfa Ordinal & 0.936 & 0.965 & 0.946 \\
Omega & 0.916 & 0.957 & 0.931 \\
Theta & 0.920 & 0.960 & 0.930 \\
$\mathrm{~N}^{\circ}$ ítems & 9 & 6 & 6 \\
\hline
\end{tabular}

Las correlaciones de Pearson entre las distintas dimensiones de la EFPA con la EFS son las esperadas (positivas y bajas). Con Estado psicológico $r=0.174(p=0.016)$; Tener Familia $r=0.297(p=0.001)$ y Logro $r=0.332(p=0.001)$. Se esperaba que no hubiera correlaciones positivas altas entre ambos instrumentos y así poder afirmar que lo que miden ambos es distinto, o que se trata de constructos fundamentalmente diferentes. Por cierto, el concepto de felicidad sobre el cual fue construida la EFPA es multidimensional, más particularmente tridimensional, en tanto el subyacente a la EFS es unidimensional, reflejando así aquél mayor diversidad, riqueza y complejidad que éste (tabla 3 ).

\section{Conclusión}

El instrumento EFPA, de origen emic y nacido en cultura chileno-latinoamericana, presenta buenas propiedades psicométricas. El instrumento captura muy confiablemente un concepto de felicidad complejo, conformado por tres subdimensiones -Equilibrio Psicológico, Satisfacción Familiar, y Orientación de Logro- que responden a lo individual la primera, a lo grupal - primario y social- la segunda, y a la relación individuo-trabajo-sociedad lo tercero. El modelo pentagonal original de felicidad sobre el cual se comenzó a construir la EFPA surgió mediante un estudio cualitativo con enfoque emic y en la publicación correspondiente se explicitó que una de ellas -Bienestares- era más bien un conjunto de condiciones necesarias o facilitadoras para ser feliz, más que un componente propiamente tal de la felicidad. Los resultados muestran que los indicadores de los modelos de tres y de cuatro factores son muy adecuados y parecidos, pero se opta por el de tres siguiendo un principio de parsimonia, además de las cuatro razones siguientes.

1. El análisis paralelo arroja una estructura tridimensional.

2. Las correlaciones inter-factores en el modelo de tres factores permiten mayor validez discriminante entre ellos (tabla 3). En el modelo de cuatro factores la correlación entre los factores Logro y Optimismo (tabla 3$)$ es demasiado alta $(r=0.867 ; p<0.01)$ lo que sugiere que comparten mucho en común y, así, son teóricamente fusionables.

3. El contenido de los ítems de las dimensión logro y optimismo de la EFPA hacen referencia ambos a la consecución de objetivos, en un tiempo presente la primera y en uno futuro la segunda.

4. En la estructura de tres factores -más que en la de cuatro- se obtiene mayor equilibrio en el número de ítems por factor.

\section{Discusión General}

El objetivo fue construir, analizar y afinar las propiedades psicométricas de la Escala de Felicidad para Adultos (EFPA), construida con base cultural de contenidos emic, para llenar un vacío en la evaluación de la felicidad en población adulta chilena y más generalmente latinoamericana. Su aplicación a una muestra final de 341 participantes permitió también verificar su estructura factorial y su correspondencia con las dimensiones del modelo teórico pentagonal de la felicidad (Moyano-Díaz, 2016a).

El instrumento EFPA presenta algunas ventajas respecto de otros para medir felicidad ya que al estar construido desde una perspectiva emic, es decir, desde los significados que participantes adultos dan al concepto de felicidad, la EFPA recoge aquellos aspectos que son sustantivos para y desde la experiencia de las personas en general, y para los chilenos en particular. Esto constituye una distinción respecto de los instrumentos anglosajones característicamente 'etic'. Adicionalmente, es una medida que considera aspectos de relevancia que asumimos son universales y diferenciados -armonía, tranquilidad, paz interior, equilibrio psicológico-, aportando más que solamente una percepción o evaluación global o inespecífica como la recogida por la EFS. En lo 
particular, la EFPA incluye aspectos específicos de alta relevancia cultural local -y más ampliamente latinoamericanacomo tener una familia, disfrutar de la misma, sentirse apoyado por ella junto con otros aspectos más universales relativos a una orientación al logro.

La EFPA presenta buenas propiedades psicométricas, lo que se corrobora mediante los distintos análisis descritos en los dos estudios. Con respecto a la evidencia de su validez de constructo el AFC produce nítidamente índices de ajuste adecuados para el modelo de tres factores. La dimensión Logro es coherente con el enfoque eudaimónico de la obtención de felicidad mediante alcanzar metas u objetivos (ojalá trascendentes), y en nuestros resultados aquella se entrecruza con optimismo, operacionalizado éste mediante ítems del tipo estilo cognitivo y disposicionales.

La EFPA no solo mostró muy buena consistencia interna, sino que mejor aún que la de la EFS (Lyubomirsky y Lepper, 1999) aplicadas ambas a las mismas personas y en mismas circunstancias. Adicionalmente, el buen ajuste del modelo de tres factores refuerza la conclusión de que los 21 ítems que constituyen la EFPA son una adecuada operacionalización de la felicidad para adultos chilenos.

Finalmente, creemos que su uso como herramienta de medida es recomendable para distintos contextos de funcionamiento -trabajo, clínico, etcétera- al permitir identificar dimensiones y aspectos específicos dentro de cada uno de ellas y, con ello, sus resultados por dimensión orientan acerca de cómo promover, recuperar o alcanzar estados emocionales o cognitivos positivos -o de agrado- en las personas.

Futuros estudios con la EFPA podrían incluir muestras de sectores populares y de fuera del valle central del territorio así como de otros países latinoamericanos para reforzar su posibilidad de aplicabilidad con confiabilidad y validez extendidas.

\section{Referencias}

Alarcón, R. (2006). Desarrollo de una escala factorial para medir la felicidad. Interamerican Journal of Psychology, 40, 95-102.

Alarcón, R. (2009). Psicología de la Felicidad: Introducción a la psicología positiva. Lima: Editorial Universitaria.

Álvarez-Ramirez, L. (2012). Escala de creencias acerca de la felicidad en población adulta de la ciudad de Bucaramanga (Colombia). Investigación y Desarrollo, 20, 303-333.

Argyle, M., Martin, M., y Crossland, J. (1989). Happiness as a function of personality and social encounters. In J. P. Forgas y J. M. Innes (Eds.), Recent advances in social psychology: An international perspective (pp. 189-203). Netherlands: Elsevier.
Árraga, M., y Sánchez, M. (2012). Validez y confiabilidad de la Escala de Felicidad de Lima en adultos mayores venezolanos. Universitas Psychologica, 11, 381-393.

Beck, A. T., Steer, R. A., y Brown, G. K. (1996). Manual for the Beck Depression Inventory-II. San Antonio, TX: Psychological Corporation.

Beck, A. T., Weissman, A., Lester, D., y Trexler, L. (1974). The measurement of pessimism: The Hopelessness Scale. Journal of Consulting and Clinical Psychology, 42, 861-865. doi:10.1037/h0037562

Bekhet, A. K., Zauszniewski, J. A., y Nakhla, W. E. (2008). Happiness: Theoretical and Empirical Considerations. Nursing Forum, 43, 12-23. doi:10.1111/j.1744-6198.2008.00091.x

Bilbao, M. A., Techio, E. M. y Páez, D. (2007). Bienestar Subjetivo, Cultura y Valores Personales: estado de la cuestión y síntesis meta-analítica de estudios. Revista de Psicología - Edición Especial en Psicología Cultural y Transcultural, 25, 233-276.

Bradburn, N. M. (1969). The structure of psychological well-being. Chicago: Aldine Pub. Co. Chicago.

Carr, A. (2004). Positive Psychology. New York: Brunner-Routledge.

Caycho, T. (2010). Variables psicológicas asociadas con la felicidad en centros peri-urbanos y urbanos marginales de Lima. UCV-Scientia, 2, 62-68.

Chekola, M.G. (1974) The concept of happiness. Unpublished PhD Dissertation, University of Michigan, Ann Arbor, USA.

Daly, M. C., y Wilson, D. J. (2009). Happiness, unhappiness, and suicide: an empirical assessment. Journal of the European Economic Association, 7, 539-549. doi:10.1162/JEEA.2009.7.2-3.539

Diener, E. (1994). Assessing subjective well-being: Progress and opportunities. Social Indicators Research, 31, 103-157. doi:10.1007/bf01207052

Diener, E., Emmons, R. A., Larsen, R. J., y Griffin, S. (1985). The Satisfaction With Life Scale. Journal of Personality Assessment, 49, 71-75. doi:10.1207/s15327752jpa4901_13

Diener, E. (2000). Subjective well-being: The science of happiness and a proposal for a national index. American Psychologist, 55, 34-43. doi:10.1037/0003-066X.55.1.34

Domínguez, S. (2012). Propuesta para el cálculo del Alfa Ordinal y Theta de Armor. Revista de Investigación en Psicología, 15, 213-2017.

Echavarrí, O., Maino, M., Fisch, R., Morales, S., y Barros, J. (2015). Aumento sostenido del suicidio en Chile: Un tema pendiente. Temas de la agenda pública, 10, 1-14.

Elosua, P., y Zumbo, B. (2008). Coeficientes de fiabilidad para escalas de respuesta categórica ordenada. Psicothema, 20, 896-901.

Fordyce, M.W. (1971). Happiness, its Daily Variation and its Relation to Values. Unpublished PhD Dissertation, U.S. International University, San Diego, California, USA.

Francis, L. J. (1999). Happiness is a thing called stable extraversion: A further examination of the relationship between the Oxford Happiness Inventory and Eysenck's dimensional model of personality and gender. Personality and Individual Differences, 26, 5-11. doi:10.1016/S01918869(98)00185-8 
Gómez, V., Villegas de Posada, C., Barrera, F., y Cruz, J. (2007). Factores predictores de bienestar subjetivo en una muestra colombiana. Revista Latinoamericana de Psicología, 39, 311-325.

Gómez, L., Alcedo, M., Arias, B., Fontanil, Y., Arias, V., Monsalve, A. y Verdugo, M.(2016). A new scale for the measurement of quality of life in children with intellectual disability. Research in Developmental disabilities, 53, 399-410. http://dx.doi.org/10.1016/j.ridd.2016.03.005

Haidt, J. (2007). The Happiness Hyphotesis: Putting ancient wisdom to the test of modern science (Vol. 1). London: Arrow Books ltd.

Hernández, K., y Muñoz, D. (2016). Felicidad y bienestar en sectores populares de la Región del Maule. Memoria para optar al título de Psicólogo. Universidad de Talca: Material no publicado.

Hills, P., y Argyle, M. (2002). The Oxford Happiness Questionnaire: a compact scale for the measurement of psychological well-being. Personality and Individual Differences, 33, 1073-1082. doi:http://dx.doi.org/10.1016/ S0191-8869(01)00213-6

Kahneman, D., Diener, E. y Schwarz, N. (Eds). (1999). Well being; the foundations of hedonic psychology. New York, Russell Sage Foundation. Kahneman, D. (2000). Experienced utility and objective happiness: a moment based approach

In: Kahneman, D. y Tverski, A. (Eds.). Choices, values and Frames. Cambridge University Press, New York.

Kahneman, D. (2011). Rápido e Devagar. Duas formas de pensar. Objetiva. Prisa Edicoes.

Kashdan, T. B., Biswas-Diener, R., y King, L. A. (2008). Reconsidering happiness: the costs of distinguishing between hedonics and eudaimonia. The Journal of Positive Psychology, 3, 219-233. doi:10.1080/17439760802303044

Kozma, A., y Stones, M. J. (1980). The measurement of happiness: development of the Memorial University of Newfoundland Scale of Happiness (MUNSH). J Gerontol, 35, 906-912.

Lyubomirsky, S., y Lepper, H. (1999). A measure of subjective happiness: Preliminary reliability and construct validation. Social Indicators Research, 46, 137-155.

Lyubomirsky, S. (2001). Why are Some People Happier than Others? The Role of Cognitive and Motivational Processes in Well-being. American Psychologist, 56, 239-249.

MacDowell, I. y Newell, C. (1996). Measuring health : a guide to rating scales and questionnaires. New York: Oxford University Press.

Mikulic, M., Cassullo, G., Crespi, M., y Marconi, A. (2009). Escala de Desesperanza BHS (A. Beck, 1974): Estudio de las propiedades psicométricas y baremización de la adaptación Argentina. Anuario de Investigaciones, 16, 365-373.

Moyano Díaz, E. (2010). Exploración de algunas propiedades psicométricas de las escalas de satisfacción vital, felicidad subjetiva y auto-percepción de salud. In E. Moyano-Díaz (Ed.), Calidad de vida y Psicología en el Bicentenario de Chile. Santiago: Mármor.
Moyano Díaz, E. (2016). Trends and Challenges for the Research of Happiness in Latin America. In M. Rojas (Ed.), Handbook of Happiness Research in Latin America (pp. 63-87). Dordrecht: Springer Netherlands.

Moyano Díaz, E. (2016a). Building the concept of happiness for adults from fenomenography. Universum, 31, 2, 141-156.

Moyano Díaz, E., Flores, E., y Soromaa, H. (2011). Fiabilidad y validez de constructo del test MUNSH para medir felicidad, en población de adultos mayores chilenos. 2010, 10, 567-580.

Moyano Díaz, E., y Ramos, N. (2007). Bienestar subjetivo: midiendo satisfacción vital, felicidad y salud en población chilena de la Región Maule. Universum (Talca), 22, 177-193.

Myers, D. G. y Diener, E. (1995). Who is Happy? Psychological Science 6, 10-19. doi: 10.1111/j.1467-9280.1995.tb00298.x

Ortiz, M. V., Gancedo, K. M., y Reyna, C. (2013). Propiedades psicométricas de la Escala de Felicidad Subjetiva en jóvenes y adultos de la ciudad de Córdoba - Argentina. Suma Psicológica, 20, 45-56. doi:10.14349/ sumapsi2013.124945-56

Pannells, T. C., y Claxton, A. F. (2008). Happiness, Creative Ideation, and Locus of Control. Creativity Research Journal, 20, 67-71. doi:10.1080/10400410701842029

Rojas, M. (2007). Heterogeneity in the relationship between income and happiness: A conceptual-referent-theory explanation. Journal of Economic Psychology, 28, 1-14. doi:http://dx.doi.org/10.1016/j.joep.2005.10.002

Rojas, M. (2016). Handbook of Happiness Research in Latin America. Netherlands: Springer

Ryan, R. M., y Deci, E. L. (2001). On Happiness and Human Potentials: A Review of Research on Hedonic and Eudaimonic Well-Being. Annual Review of Psychology, 52, 141-166. doi:doi:10.1146/annurev. psych.52.1.141

Scalco, D. L., Araújo, C. L., y Bastos, J. L. (2011). Autopercepção de felicidade e fatores associados em adultos de uma cidade do sul do Brasil: estudo de base populacional. Psicologia: Reflexão e Crítica, 24(4), 648-657.

Schermelleh-Engel K, Moosbrugger, H., y Müller, H. (2003). Evaluating the Fit of Structural Equation Models: Tests of Significance and Descriptive Goodness-of-Fit Measures. Methods of Psychological Research Online, 8, 23-74.

Scorsolini-Comin, F., y dos Santos, M. (2010). El estudio científico de la felicidad y la promoción de la salud: revisión integradora de la literatura. Revista Latinoamericana de Enfermagem, 18, 192-199.

Seligman, M. (2004). Felicidade autêntica: usando a nova Psicologia Positiva para a realização permanente. Rio de Janeiro: Objetiva.

Shin, D. y Johnson, D. M. (1978) Avowed happiness as the overall assessment of the quality of life. Social Indicators Research, 5, 475492. doi:10.1007/bf00352944

Silva-Colmenares, J. (2008). Felicidad: la evolución como categoría científica y la relación con el desarrollo. Revista de la información Básica CANDANE (Bogotá), 3, 62-77. 
Stewart, M. E., Watson, R., Clark, A., Ebmeier, K. P., y Deary, I. J. (2010). A hierarchy of happiness? Mokken scaling analysis of the Oxford Happiness Inventory. Personality and Individual Differences, 48, 845848. doi:http://dx.doi.org/10.1016/j.paid.2010.02.011

Tkach, C. y Lyubomirsky, S. (2006). How do people pursue happiness?: relating personality, happiness-increasing strategies, and well-being. Journal of Happiness Studies, 7, 183-225. doi:10.1007/s10902-005-4754-1

Tornimbeni, S., Pérez, E., y Olaz, F. (2008). Introducción a la Psicometría. Argentina: Paidós.

Veenhoven, R. (1994). Is happiness a trait? Tests of the theory that a better society does not make people any happier. Social Indicators Research, 32, 101-160. doi:10.1007/bf01078732
Veenhoven, R. (2006). How do we assess how happy we are? Tenets, implications and tenability of three theories. Paper presented at conference on 'New Directions in the Study of Happiness: United States and International Perspectives', University of Notre Dame, USA, October 22-24 2006

Vera-Villarroel, P., Córdova-Rubio, N., y Celis-Atenas, K. (2009). Evaluación del optimismo: un análisis preliminar del Life Orientation Test versión revisada (LOT-R) en población chilena. Universitas Psychologica, 8, 61-68.

Vera-Villarroel, P., Celis-Atenas, K., y Córdova-Rubio, N. (2011). Evaluacion de la felicidad: análisis psicométrico de la Escala de Felicidad Subjetiva en población chilena. Terapia Psicológica, 29, 127-133. 
